\title{
Adverse events associated with incretin-based drugs in japanese spontaneous reports: a mixed effects logistic regression model
}

Daichi Narushima, Yohei Kawasaki, Shoji Takamatsu, Hiroshi Yamada

Background: Spontaneous reporting systems (SRSs) are passive systems composed of reports of suspected adverse drug events (ADEs), and are used for pharmacovigilance (PhV), namely, drug safety surveillance. Exploration of analytical methodologies to enhance SRS-based discovery will contribute to more effective PhV. In this study, we proposed a statistical modeling approach for SRS data to address heterogeneity by a reporting time point. Furthermore, we applied this approach to analyze ADEs of incretinbased drugs such as DPP-4 inhibitors and GLP-1 receptor agonists, which are widely used to treat type 2 diabetes. Methods: SRS data were obtained from the Japanese Adverse Drug Event Report (JADER) database. Reported adverse events were classified according to the MedDRA High Level Terms (HLTs). A mixed effects logistic regression model was used to analyze the occurrence of each HLT. The model treated DPP-4 inhibitors, GLP-1 receptor agonists, hypoglycemic drugs, concomitant suspected drugs, age, and sex as fixed effects, while the quarterly period of reporting was treated as a random effect. Before applications of the model, Fisher's exact tests were performed for all drug-HLT combinations. Mixed effects logistic regressions were performed for the HLTs that were found to be associated with incretin-based drugs. Statistical significance was determined by a two-sided p-value $<0.01$ or a $99 \%$ two-sided confidence interval. Finally, the models with and without the random effect were compared based on AIC (Akaike's information criteria), in which a model with a smaller AIC was considered satisfactory. Results: The analysis included 187,181 cases reported from January 2010 to March 2015. It showed that 33 HLTs, including pancreatic, gastrointestinal, and cholecystic events, were significantly associated with DPP-4 inhibitors or GLP-1 receptor agonists. In the AIC comparison, half of the HLTs reported with incretin-based drugs favored the random effect, whereas HLTS reported frequently tended to favor the mixed model. Conclusion: The model with the random effect was appropriate for analyzing frequently reported ADEs; however, further exploration is required to improve the model. The core concept of the model is to introduce a random effect of time. Modeling the random effect of time is widely applicable to various SRS data and will improve future SRS data analyses. 


\section{Adverse Events Associated with Incretin-based Drugs in Japanese} 2 Spontaneous Reports: A Mixed Effects Logistic Regression Model

3

4 Daichi Narushima ${ }^{1}$, Yohei Kawasaki ${ }^{1}$, Shoji Takamatsu ${ }^{2}$, Hiroshi Yamada ${ }^{1}$

$5 \quad{ }^{1}$ Department of Drug Evaluation \& Informatics, Graduate school of Pharmaceutical Sciences, 6 University of Shizuoka, Shizuoka, Japan

$7 \quad{ }^{2}$ Office of Safety II, Pharmaceuticals and Medical Devices Agency, Japan

8 Corresponding author:

9 Hiroshi Yamada, MD, PhD, FACP

10 Department of Drug Evaluation \& Informatics, Graduate School of Pharmaceutical Sciences, 11 University of Shizuoka, 52-1 Yada, Suruga-ku, Shizuoka 422-8526, Japan

12 Email: hyamada@u-shizuoka-ken.ac.jp 


\section{Abstract}

15 Background

16 Spontaneous reporting systems (SRSs) are passive systems composed of reports of suspected 17 adverse drug events (ADEs), and are used for pharmacovigilance ( $\mathrm{PhV}$ ), namely, drug safety 18 surveillance. Exploration of analytical methodologies to enhance SRS-based discovery will 19 contribute to more effective $\mathrm{PhV}$. In this study, we proposed a statistical modeling approach for 20 SRS data to address heterogeneity by a reporting time point. Furthermore, we applied this 21 approach to analyze ADEs of incretin-based drugs such as DPP-4 inhibitors and GLP-1 receptor 22 agonists, which are widely used to treat type 2 diabetes.

\section{Methods}

24 SRS data were obtained from the Japanese Adverse Drug Event Report (JADER) database. 25 Reported adverse events were classified according to the MedDRA High Level Terms (HLTs). A mixed effects logistic regression model was used to analyze the occurrence of each HLT. The model treated DPP-4 inhibitors, GLP-1 receptor agonists, hypoglycemic drugs, concomitant suspected drugs, age, and sex as fixed effects, while the quarterly period of reporting was treated as a random effect. Before applications of the model, Fisher's exact tests were performed for all drug-HLT combinations. Mixed effects logistic regressions were performed for the HLTs that were found to be associated with incretin-based drugs. Statistical significance was determined by a two-sided p-value $<0.01$ or a $99 \%$ two-sided confidence interval. Finally, the models with and without the random effect were compared based on AIC (Akaike's information criteria), in which a model with a smaller AIC was considered satisfactory.

Results

The analysis included 187,181 cases reported from January 2010 to March 2015. It showed that 33 HLTs, including pancreatic, gastrointestinal, and cholecystic events, were significantly associated with DPP-4 inhibitors or GLP-1 receptor agonists. In the AIC comparison, half of the HLTs reported with incretin-based drugs favored the random effect, whereas HLTs reported frequently tended to favor the mixed model.

41 Conclusion

42 The model with the random effect was appropriate for analyzing frequently reported ADEs; however, further exploration is required to improve the model. The core concept of the model is to introduce a random effect of time. Modeling the random effect of time is widely applicable to various SRS data and will improve future SRS data analyses. 


\section{Introduction}

48

49

50

51

52

53

54

55

56

57

58

59

60

61

62

63

64

65

66

67

68

69

70

71

72

73

74

75

76

77

78

79

80

81

82

83

84

85

86

87 88

The incretins are a group of intestinal hormones that stimulate insulin secretion. During the last decade, several hypoglycemic drugs based on incretin have gained widespread use as treatments for patients with type 2 diabetes. Incretin-based drugs are classified as inhibitors of incretindegrading protease dipeptidyl peptidase 4 (DPP-4) or as incretin hormone glucagon-like peptide 1 (GLP-1) receptor agonists. DPP-4 inhibitors and GLP-1 receptor agonists lower fasting and postprandial glucose, but do not produce hypoglycemia and are not associated with body weight gain or reduced in blood pressure. (Nauck 2013)

DPP-4 inhibitors and GLP-1 receptor agonists have been associated with adverse outcomes, including pancreatic disorders, although some of these findings are controversial. (Butler et al. 2013; Devaraj \& Maitra 2014; Nauck 2013) An analysis of the US Food and Drug Administration (FDA) Adverse Event Reporting System (FAERS) revealed that use of DPP-4 inhibitor sitagliptin or GLP-1 receptor agonist exenatide increased the odds ratio (OR) for pancreatitis more than 6-fold, while increasing the OR for pancreatic cancer more than 2-fold, in comparison with other medications; (Elashoff et al. 2011) however, most other clinical studies have demonstrated no evidence suggesting such risks. (Li et al. 2014; Nauck 2013) The FDA and European Medicines Agency (EMA) explored multiple streams of data and agreed that assertions concerning a causal association between incretin-based drugs and pancreatitis or pancreatic cancer were inconsistent with the current data. (Egan et al. 2014)

Spontaneous reporting systems (SRSs) such as the FAERS are passive systems composed of reports of suspected adverse drug events (ADEs) collected from healthcare professionals, consumers, and pharmaceutical companies. (Harpaz et al. 2012) SRSs play an essential role in pharmacovigilance $(\mathrm{PhV})$, which is also referred to as drug safety surveillance. Although SRSs cover large populations, their data have some biases in reporting. In the case of incretin-based drugs in the FAERS, reporting of pancreatitis was largely influenced by the relevant FDA alerts in an example of notoriety bias, which could cause overestimation of risk. (Raschi et al. 2013) Spontaneous reporting patterns change over time, which is one of the limitations of SRSs. (Bate \& Evans 2009) SRSs have numerous limitations; nevertheless, PhV has relied predominantly on SRSs. (Gibbons et al. 2010; Harpaz et al. 2012) Therefore, exploration of novel analytical methodologies to enhance SRS-based discovery will highlight the value of SRSs and contribute to more effective $\mathrm{PhV}$.

The most conventional analytical approach for SRS data is disproportionality analyses (DPA). DPA methodologies use frequency analysis of $2 \times 2$ contingency tables to quantify the degree to which a drug-event combination co-occurs disproportionately in comparison with that expected in the absence of an association. (Bate \& Evans 2009; Harpaz et al. 2013) DPA do not require complicated modeling; however, these do not adjust for confounding factors. The alternatives to DPA are multivariate modeling techniques such as logistic regression. Multivariate modeling techniques can adjust for potential confounding and masking factors during the analysis of drugevent relationships. (Harpaz et al. 2013) In a performance evaluation using the FAERS, approaches based on logistic regression outperformed DPA approaches. (Harpaz et al. 2013)

The objectives of the present study are to propose a novel multivariate modeling approach for SRS data and to apply this approach to analyze ADEs associated with incretin-based drugs from 
89

90

91

92

93

94

95

96

97

98

99

100

101

102

103

104

105

106

107

108

109

110

111

112

113

114

115

116

117

118

119

120

121

122

123

124

125

126

127

128

an SRS. We designed a mixed effects logistic regression model and performed comprehensive analyses using this model. The analyzed data were obtained from the Japanese Adverse Drug Event Report (JADER) database maintained by the Pharmaceuticals and Medical Devices Agency (PMDA). (PMDA 2015) Most case reports in the FAERS are from consumers or lawyers, whereas those in the JADER are medically confirmed. (Nomura et al. 2015) The analyses were based mainly on mixed effects logistic regression, in which DPA were used adjunctively. Mixed effects logistic regression models contain variables for random effects in addition to those for fixed effects similar to conventional logistic regression models. The random variable in a logistic regression model describes the ramifications of different sources of heterogeneity and associations between outcomes. (Larsen et al. 2000) Mixed effects logistic regression model is a type of generalized linear mixed models (GLMMs). The use of GLMMs in medical literature has recently increased to take into account data correlations when modeling binary or count data; (Casals et al. 2014) however, applications of GLMMs to SRS data have been reported rarely. As a rare application of GLMMs to SRS data, an approach based on a mixed effects Poisson regression model was proposed. (Gibbons et al. 2008) This approach yields rate multipliers for each drug in a class of drugs, which describe the deviation of the rate for a specific adverse event from that for the drug class as a whole. In contrast, the present approach is based on a logistic regression model with a random effect of time and addresses heterogeneity between time points. Since reporting-time-point is an attribute common to SRSs, modeling the random effect of time is widely applicable to any ADE in any SRS. Nevertheless, to the best of our knowledge, modeling approaches such as this have not been reported. This is the first application of a logistic regression model with a random effect of time to SRS data.

\section{Material and Methods}

\section{Data Source}

The JADER dataset, which was published in July 2015 and contained 353,988 unique cases, was obtained from the website of the PMDA. The analyzed cases were reported from January 2010 to March 2015 and had available records regarding age and sex.

Adverse events in the JADER were coded as "Preferred Terms" (PTs) in the Japanese version of the Medical Dictionary for Regulatory Activities (MedDRA/J). (MedDRA 2015) MedDRA has a hierarchical structure, in which PTs are grouped into "High Level Terms" (HLTs), HLTs are grouped into "High Level Group Terms" (HLGTs), and HLGTs are grouped into "System Organ Classes" (SOCs). Before data analyses, a relational database was constructed from the JADER dataset and MedDRA/J version 18.0. SQLite version 3.8.5 was used as the database management system. (SQLite Development Team 2015)

As incretin-based drugs, all DPP-4 inhibitors and GLP-1 receptor agonists approved in Japan were assessed. The DPP-4 inhibitors were sitagliptin phosphate hydrate, vildagliptin, alogliptin benzoate, alogliptin benzoate/pioglitazone hydrochloride (combination drug), linagliptin, teneligliptin hydrobromide hydrate, anagliptin, and saxagliptin hydrate. The GLP-1 receptor agonists were exenatide, liraglutide, and lixisenatide.

\section{Data Analysis}


129 The analysis of ADEs associated with incretin-based drugs was composed of two phases. The

130 first phase was a DPA based on Fisher's exact test. The second phase was a multivariate analysis

131 using a mixed effects logistic regression model.

132 The PTs of ADEs were classified according to the MedDRA HLTs. All combinations of generic 133 drug names and HLTs were extracted. Fisher's exact tests were performed for all combinations of 134 incretin-based drugs and reported HLTs. Associations that yielded a two-sided p-value $<0.01$ and 135 an OR $>1$ were considered significant.

136 Mixed effects logistic regressions were performed for each HLT significantly associated with incretin-based drugs. The mixed effects logistic regression model was as follows:

$$
\frac{P\left(Y_{i}=1 \mid x_{i}, z_{i}\right)}{P\left(Y_{i}=0 \mid x_{i}, z_{i}\right)}=\exp \left(x_{i}^{T} \beta+z_{i}^{T} u\right)
$$

Where $Y_{i}$ is a binary variable describing the outcome of case $i$ ( 0 or 1$), \beta$ is a fixed parameter vector, $x_{i}$ is a covariate vector for fixed effects, $u$ is a vector of random variables from probability distributions, and $z_{i}$ is a covariate vector for random effects. $u$ represents unmeasured covariates as a way of modeling heterogeneity and correlated data. (Larsen et al. 2000)

In the newly developed model, the binary outcome was whether or not each HLT was reported. For fixed effects, the covariates were use of DPP-4 inhibitors, use of GLP-1 receptor agonists, use of any hypoglycemic drugs (an alternative indicator for hyperglycemia), sum of concomitant suspected drugs (determined by reference to the Fisher's exact tests), age (in 10-year intervals), and sex. The random effect was the quarterly period of reporting. The variables for the random effect were random intercepts normally distributed with mean 0 and one common variance. The associations between incretin-based drugs and HLTs were assessed by ORs with $99 \%$ two-sided Wald-type confidence intervals (CIs). Because the present analysis was an exploratory screening, the problem of multiple comparison was not addressed. Instead of correcting that, stringent levels of statistical significance were set ( $\mathrm{p}$-value $<0.01$ and $99 \% \mathrm{CI}$ ).

153 The newly developed mixed model was compared with a fixed model that did not include the random effect. The covariates for fixed effects in the fixed model were the same covariates use in the mixed model. Logistic regressions based on each model were performed for all reported HLTs associated with incretin-based drugs. Subsequently, the adequacy of the model was assessed by AIC (Akaike's information criteria). (Burnham \& Anderson 2002) A model with a smaller AIC was favored.

All analyses were performed using the $\mathrm{R}$ version 3.2.1. (R Core Team 2015) The glmmML package version 1.0 was used with the "ghq" (Gauss-Hermite quadrature) method for the mixed

\section{Results}

163 Description of the analyzed case reports

164 The JADER included 204,472 unique cases that were reported from January 2010 to March 1652015 , of which 187,181 had available records for age and sex and were analyzed. The records 166 included 4,952 generic drug names and 6,151 PTs under 1,377 HLTs. DPP-4 inhibitors were 
167 mentioned in 7,265 cases, whereas GLP-1 receptor agonists were mentioned in 451 cases. Figure

1681 shows the number of cases mentioning hypoglycemic drugs that were reported during each

169 quarterly period. Although the number of cases for other hypoglycemic drugs increased

170 gradually over time, the number of cases for DPP-4 inhibitors increased markedly.

171 Mixed effects logistic regressions

172 The cases associated with incretin-based drugs included 1,430 PTs under 735 HLTs. The Fisher's 173 exact tests showed that 106 of the 735 HLTs were significantly associated with any incretin-

174 based drug (two-sided $p$-value $<0.01$ and OR $>1$ ). In the mixed effects logistic regressions, 33 of

175 the 106 HLTs identified by the Fisher's exact tests were significantly associated with DPP-4

176 inhibitors or GLP-1 receptor agonists (99\% two-sided CI). Table 1 shows the number of cases

177 reported for each HLT. Figure 2 shows ORs with 99\% CIs for the significant associations

178 between HLTs and DPP-4 inhibitors or GLP-1 receptor agonists. "NEC" in the MedDRA terms

179 is an acronym for "Not Elsewhere Classified", which denotes groupings of miscellaneous terms,

180 whereas "excl" is an abbreviation of "excluding". The HLTs associated with DPP-4 inhibitors

181 included "Pancreatic disorders NEC" (OR 18.66; 99\% CI 2.09-166.25) and "Acute and chronic

182 pancreatitis" $(8.65 ; 5.76-12.98)$. The HLTs associated with GLP-1 receptor agonists included

183 "Thyroid neoplasms" (87.25; 6.64-1146.27) and "Cystic pancreatic disorders" (61.32; 1.69 -

184 2224.49). The HLTs associated with DPP-4 inhibitors and GLP-1 receptor agonists indicated

185 pancreatic events ("Acute and chronic pancreatitis", "Pancreatic neoplasms", "Pancreatic

186 neoplasms malignant (excl islet cell and carcinoid)", and "Pancreatic disorders NEC"),

187 gastrointestinal events ("Benign neoplasms gastrointestinal (excl oral cavity)" and

188 "Gastrointestinal stenosis and obstruction NEC"), and cholecystic events ("Cholecystitis and

189 cholelithiasis"). Although DPP-4 inhibitors and GLP-1 receptor agonists were not associated

190 with hypoglycemic events, GLP-1 receptor agonists were associated with several HLTs related

191 to diabetes, including "Hyperglycaemic conditions NEC" and "Diabetic complications NEC".

192 Comparison between the models with and without the random effect

193 Figure 3 shows the comparison between the models with (mixed model) and without (fixed

194 model) the random effect. In 604 of the 735 HLTs reported with incretin-based drugs, the AIC of

195 the models were calculated normally. Of the 604 HLTs, 302 favored the mixed model, whereas

196 the others favored the fixed model. The median number of reported cases for the group of HLTs

197 favoring the mixed model was 264, whereas the median number of reported cases for the group

198 of HLTs favoring the fixed model was 83; thus, HLTs reported frequently tended to favor the

199 mixed model.

\section{Discussion}

201 Time-series variation of spontaneous reports

202 SRSs accumulate a large amount of data regarding ADEs every year; thus, the contents of SRSs

203 are not constant. In the present study, the report composition of hypoglycemic drug groups

204 varied during the study period. Reports associated with DPP-4 inhibitors showed a marked

205 increase in comparison with that of reports associated with other hypoglycemic drugs, perhaps

206 because of an increasing number of approved products and an associated increase in drug use.

207 The number of reports during a particular period is affected by numerous factors. This variation 
208 in reporting results in temporal heterogeneity, supporting the appropriateness of the mixed 209 model.

210 Adverse events associated with incretin-based drugs

211 Some HLTs associated with incretin-based drugs in the present study have been reported as 212 issues of concern in previous studies. Some groups of similar HLTs, e.g., "Thyroid neoplasms" 213 and "Thyroid neoplasms malignant" were identified because some PTs are linked to multiple 214 HLTs in the MedDRA. In comparison with DPP-4 inhibitors, GLP-1 receptor agonists showed 215 relatively wide CIs for some of the HLTs because fewer cases were reported, leading to 216 unreliable results.

217 In the present study, pancreatic disorders, including pancreatitis and pancreatic cancer, were 218 associated with DPP-4 inhibitors and GLP-1 receptor agonists, which were consistent with 219 results obtained via analysis of the FAERS. (Butler et al. 2013; Elashoff et al. 2011) In addition, 220 thyroid cancer was associated with GLP-1 receptor agonists; however, because of the small 221 number of cases, this finding is unreliable. Analyses of the FAERS also indicated that GLP-1 222 receptor agonists increased ORs for thyroid cancer. (Butler et al. 2013; Elashoff et al. 2011) Thyroid cancer and pancreatic disorders are among the most controversial safety concerns regarding incretin-based drugs; however, no evidence has been found for such risks in human clinical studies. (Butler et al. 2013; Nauck 2013) The other HLTs associated with DPP-4 inhibitors and GLP-1 receptor agonists were "Benign neoplasms gastrointestinal (excl oral cavity)", "Gastrointestinal stenosis and obstruction NEC", and "Cholecystitis and cholelithiasis". Gastrointestinal events such as nausea, vomiting, and diarrhea are common ADEs of incretinbased drugs; (Nauck 2011) however, benign gastrointestinal neoplasms, stenosis, and obstruction have not been referred to in past studies. Cholecystic events have not in the same way.

231 Hypoglycemia, an adverse event associated with some hypoglycemic drugs, was not associated with incretin-based drugs. In contrast, hyperglycemia and several other diabetic complications were associated with GLP-1 receptor agonists, perhaps because of cases of ineffective drug treatment.

\section{Limitations}

236

237

238

239

240

241

242

243

244

245

246

247 Mixed effects logistic regression model 
248 2

In the AIC comparison between the mixed model and the fixed model, half of the HLTs reported with incretin-based drugs favored each model. The HLTs that favored the mixed model were reported more frequently than those that favored the fixed model, indicating that the mixed model may be more appropriate for frequently reported ADEs. The AIC formula has a biascorrection term for the number of estimable parameters. (Burnham \& Anderson 2002) In the above comparison, the mixed model has only one more parameter than does the fixed model; hence, the difference between the penalties for the correction is small.

The adequacy of the random effect was demonstrated; however, the model can be improved. Although we assumed a normal distribution for the random effect, the appropriateness of this assumption is unclear. Moreover, it is unclear whether a single probability distribution is sufficient to assess the random effect on the widely spread time-scale of spontaneous reports. Sampling of parameter distributions by Bayesian hierarchical modeling is a potential solution to these problems. Currently, diverse implementations of Bayesian methods, which support practice of such modeling, are accessible. (Li et al. 2011; MacLehose \& Hamra 2014)

\section{Conclusion}

We proposed a logistic regression model for SRS data taking into account the random effect of time and applied this model to analyze ADEs reported with incretin-based drugs in the JADER. The newly developed model was appropriate for ADEs reported frequently; however, further exploration is required to improve the model.

The core concept of the newly developed model is to introduce a random effect of time. The random effect introduced into a univariate or multivariate model addresses heterogeneity by time unit. Reporting-time-point is an attribute common to all spontaneous reports. Hence, modeling the random effect of time is widely applicable to various SRS data and will improve future statistical analyses of SRS data.

.

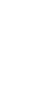




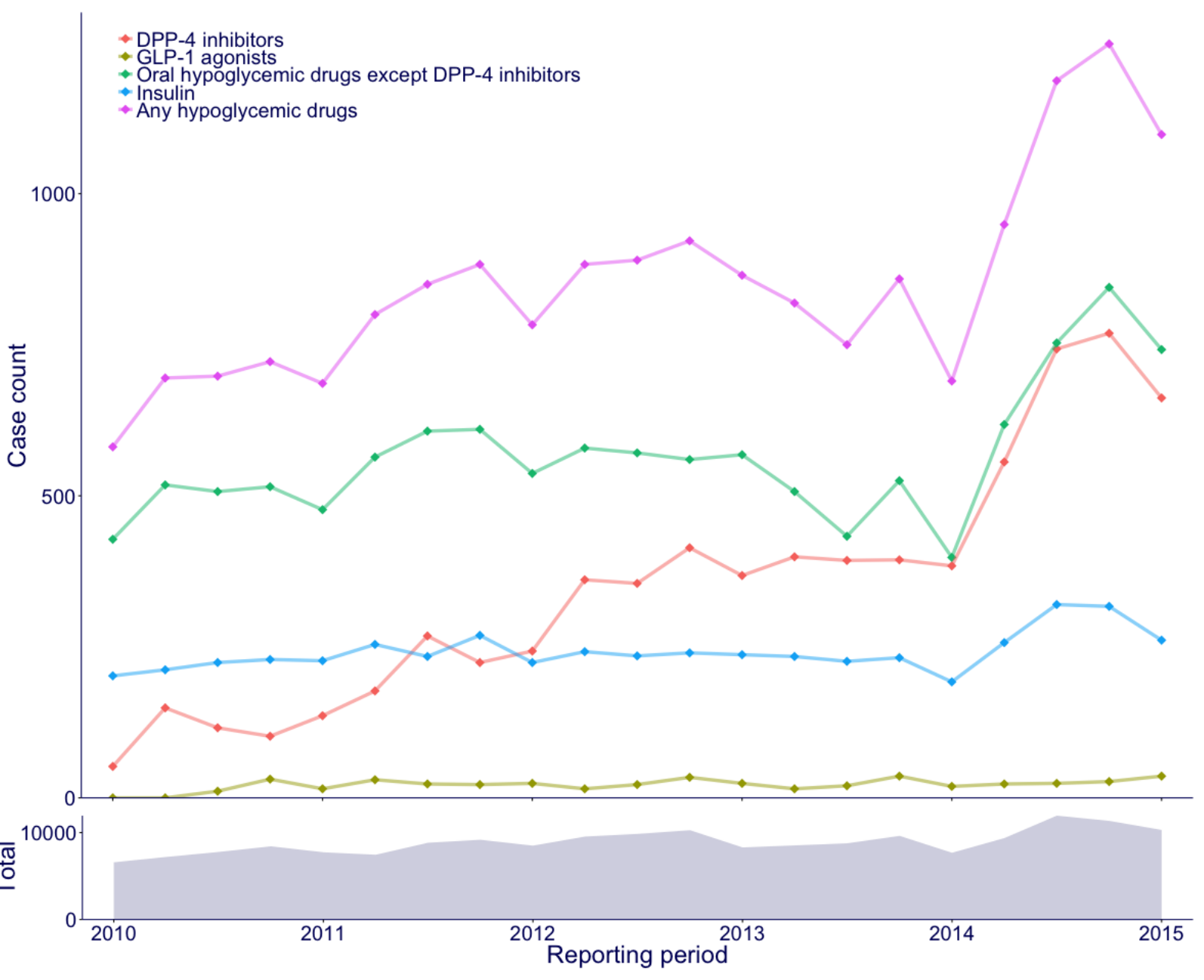

278 Figure 1 Case counts of hypoglycemic drugs by each quarterly period.

279 The upper line plot denotes cases reported with hypoglycemic drugs. The lower area plot denotes 280 all cases. 


\begin{tabular}{|c|c|c|c|}
\hline MedDRA HLT & $\begin{array}{c}\text { DPP-4 } \\
\text { inhibitors }\end{array}$ & $\begin{array}{l}\text { GLP-1 receptor } \\
\text { agonists }\end{array}$ & Total \\
\hline Thyroid neoplasms & 1 & 3 & 62 \\
\hline Thyroid neoplasms malignant & 0 & 2 & 53 \\
\hline Cystic pancreatic disorders & 2 & 1 & 16 \\
\hline Pancreatic disorders NEC & 11 & 3 & 50 \\
\hline Adrenal cortical hypofunctions & 5 & 4 & 184 \\
\hline Gastrointestinal neoplasms benign NEC & 5 & 2 & 22 \\
\hline Chronic polyneuropathies & 3 & 2 & 44 \\
\hline Pancreatic neoplasms & 47 & 16 & 166 \\
\hline Cholecystitis and cholelithiasis & 39 & 12 & 441 \\
\hline Bile duct infections and inflammations & 9 & 4 & 176 \\
\hline Pancreatic neoplasms malignant (excl islet cell and carcinoid) & 42 & 13 & 142 \\
\hline Injection site reactions & 6 & 8 & 742 \\
\hline Non-mechanical ileus & 16 & 7 & 325 \\
\hline Diabetic complications NEC & 23 & 19 & 177 \\
\hline Acute and chronic pancreatitis & 234 & 29 & 1038 \\
\hline Gastrointestinal atonic and hypomotility disorders NEC & 20 & 9 & 390 \\
\hline Gastric neoplasms malignant & 19 & 5 & 279 \\
\hline Benign neoplasms gastrointestinal (excl oral cavity) & 16 & 3 & 95 \\
\hline Skin autoimmune disorders NEC & 27 & 0 & 186 \\
\hline Rheumatoid arthropathies & 17 & 1 & 153 \\
\hline Rheumatoid arthritis and associated conditions & 17 & 1 & 154 \\
\hline Hyperglycaemic conditions NEC & 92 & 34 & 728 \\
\hline Arthropathies NEC & 24 & 0 & 417 \\
\hline Lower respiratory tract neoplasms & 26 & 4 & 393 \\
\hline Lower gastrointestinal neoplasms benign & 10 & 2 & 51 \\
\hline Diabetic complications neurological & 15 & 4 & 71 \\
\hline Gastrointestinal stenosis and obstruction NEC & 114 & 11 & 1216 \\
\hline Urinalysis NEC & 36 & 1 & 149 \\
\hline Digestive enzymes & 23 & 2 & 249 \\
\hline Metabolic acidoses (excl diabetic acidoses) & 98 & 14 & 611 \\
\hline Skeletal and cardiac muscle analyses & 66 & 1 & 896 \\
\hline Non-site specific injuries NEC & 76 & 4 & 1179 \\
\hline Coronary necrosis and vascular insufficiency & 141 & 12 & 1555 \\
\hline
\end{tabular}

284 Table 1 Case counts of adverse events associated with DPP-4 inhibitors or GLP-1 receptor 285 agonists. 
Pancreatic neoplasms malignant (excl islet cell and carcinoid). Injection site reactions

Non-mechanical ileus Diabetic complications NEC

Acute and chronic pancreatitis Gastrointestinal atonic and hypomotility disorders NEC

Gastric neoplasms malignant Benign neoplasms gastrointestinal (excl oral cavity)

Skin autoimmune disorders NEC

Rheumatoid arthropathies

Rheumatoid arthritis and associated conditions

Hyperglycaemic conditions NEC

Arthropathies NEC

Lower respiratory tract neoplasms

Lower gastrointestinal neoplasms benign

Diabetic complications neurological Gastrointestinal stenosis and obstruction NEC Urinalysis NEC

Digestive enzymes

Metabolic acidoses (excl diabetic acidoses).

Skeletal and cardiac muscle analyses

Non-site specific injuries NEC

Coronary necrosis and vascular insufficiency

DPP-4 inhibitors

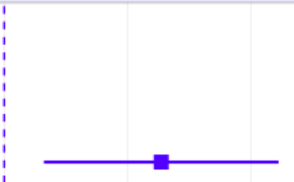

$\rightarrow$
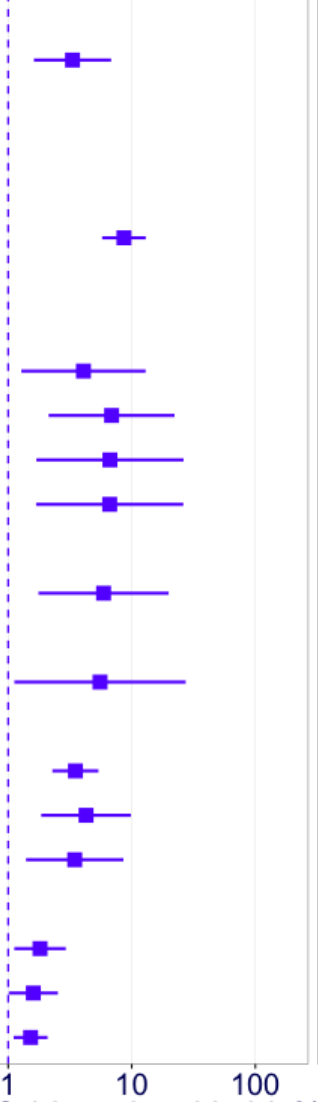

Odds ratio with $99 \%$ confidence interval

291 Figure 2 Odds ratios of the adverse events associated with DPP-4 inhibitors or GLP-1 receptor 292 agonists.

293 The forest plot denotes odds ratios (ORs) with 99\% confidence intervals (CIs) for each event. 294 Significant ORs with CIs are plotted. 


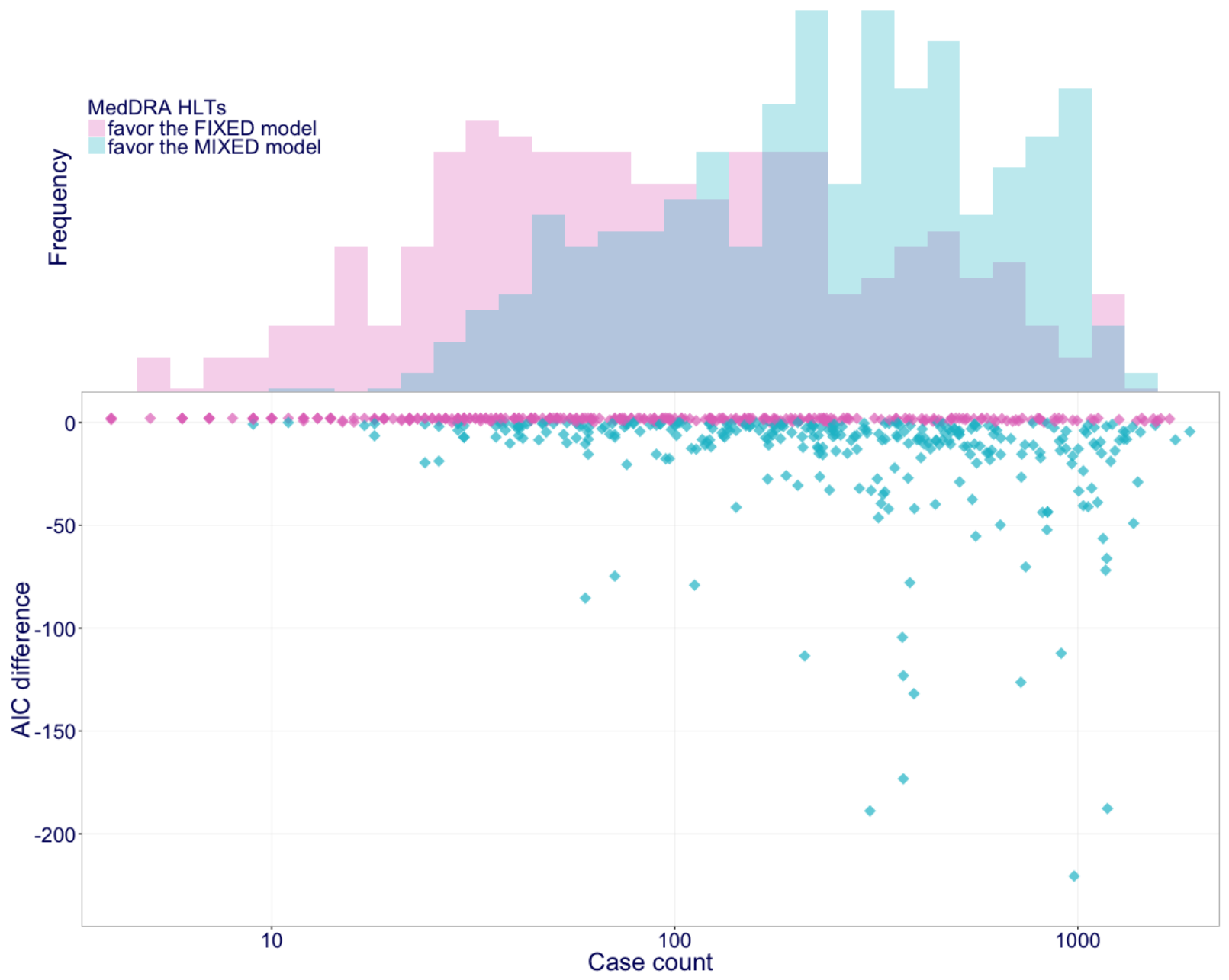

298

299 Figure 3 AIC improvements with a random effect.

300 The vertical axis of the lower scatter plot denotes AIC differences calculated by subtracting that 301 of the fixed model from that of the mixed model. When the AIC difference is less than 0 , the 302 mixed model is favored. The horizontal axis denotes total case counts for each MedDRA HLT. 303 The upper plot is the histogram of the lower plot. 
305

306

307

308

309

310

311

312

313

314

315

316

317

318

319

320

321

322

323

324

325

326

327

328

329

330

331

332

333

334

335

336

337

338

339

340

341

342

343

344

345

346

347

348

\section{References}

Bate A, and Evans SJ. 2009. Quantitative signal detection using spontaneous ADR reporting. Pharmacoepidemiol Drug Saf 18:427-436. 10.1002/pds.1742

Brewer T, and Colditz GA. 1999. Postmarketing surveillance and adverse drug reactions: current perspectives and future needs. JAMA 281:824-829. 10.1001/jama.281.9.824

Broström G. 2013. glmmML: Generalized linear models with clustering.

Burnham KP, and Anderson DR. 2002. Model selection and multimodel inference: a practical information-theoretic approach: Springer Science \& Business Media.

Butler PC, Elashoff M, Elashoff R, and Gale EA. 2013. A critical analysis of the clinical use of incretin-based therapies: Are the GLP-1 therapies safe? Diabetes Care 36:21182125. $10.2337 / \mathrm{dc} 12-2713$

Casals M, Girabent-Farres M, and Carrasco JL. 2014. Methodological quality and reporting of generalized linear mixed models in clinical medicine (2000-2012): a systematic review. PLoS One 9:e112653. 10.1371/journal.pone.0112653

Devaraj S, and Maitra A. 2014. Pancreatic safety of newer incretin-based therapies: are the "-tides" finally turning? Diabetes 63:2219-2221. 10.2337/db14-0545

Egan AG, Blind E, Dunder K, de Graeff PA, Hummer BT, Bourcier T, and Rosebraugh C. 2014. Pancreatic safety of incretin-based drugs--FDA and EMA assessment. $N$ Engl J Med 370:794-797. 10.1056/NEJMp1314078

Elashoff M, Matveyenko AV, Gier B, Elashoff R, and Butler PC. 2011. Pancreatitis, pancreatic, and thyroid cancer with glucagon-like peptide-1-based therapies. Gastroenterology 141:150-156. 10.1053/j.gastro.2011.02.018

Gibbons RD, Amatya AK, Brown CH, Hur K, Marcus SM, Bhaumik DK, and Mann JJ. 2010. Post-approval drug safety surveillance. Annu Rev Public Health 31:419-437. 10.1146/annurev.publhealth.012809.103649

Gibbons RD, Segawa E, Karabatsos G, Amatya AK, Bhaumik DK, Brown CH, Kapur K, Marcus SM, Hur K, and Mann JJ. 2008. Mixed-effects Poisson regression analysis of adverse event reports: the relationship between antidepressants and suicide. Stat Med 27:1814-1833. 10.1002/sim.3241

Harpaz R, DuMouchel W, LePendu P, Bauer-Mehren A, Ryan P, and Shah NH. 2013. Performance of pharmacovigilance signal-detection algorithms for the FDA adverse event reporting system. Clin Pharmacol Ther 93:539-546. 10.1038/clpt.2013.24

Harpaz R, DuMouchel W, Shah NH, Madigan D, Ryan P, and Friedman C. 2012. Novel datamining methodologies for adverse drug event discovery and analysis. Clin Pharmacol Ther 91:1010-1021. 10.1038/clpt.2012.50

Larsen K, Petersen JH, Budtz-Jorgensen E, and Endahl L. 2000. Interpreting parameters in the logistic regression model with random effects. Biometrics 56:909-914. 10.1111/j.0006-341X.2000.00909.x

Li B, Lingsma HF, Steyerberg EW, and Lesaffre E. 2011. Logistic random effects regression models: a comparison of statistical packages for binary and ordinal outcomes. BMC Med Res Methodol 11:77. 10.1186/1471-2288-11-77

Li L, Shen J, Bala MM, Busse JW, Ebrahim S, Vandvik PO, Rios LP, Malaga G, Wong E, Sohani Z, Guyatt GH, and Sun X. 2014. Incretin treatment and risk of pancreatitis in patients 
355

356

357

358

359

360

361

362

363

364

365

366

367

368

369

370

371

372

373

with type 2 diabetes mellitus: systematic review and meta-analysis of randomised and non-randomised studies. BMJ 348:g2366. 10.1136/bmj.g2366

MacLehose RF, and Hamra GB. 2014. Applications of Bayesian Methods to Epidemiologic Research. Current Epidemiology Reports 1:103-109. 10.1007/s40471-014-0019-z MedDRA. 2015. MedDRA. Available at http://www.meddra.org/.

Nauck MA. 2011. Incretin-based therapies for type 2 diabetes mellitus: properties, functions, and clinical implications. Am J Med 124:S3-18. 10.1016/j.amjmed.2010.11.002

Nauck MA. 2013. A critical analysis of the clinical use of incretin-based therapies: The benefits by far outweigh the potential risks. Diabetes Care 36:2126-2132. $10.2337 / \mathrm{dc} 12-2504$

Nomura K, Takahashi K, Hinomura Y, Kawaguchi G, Matsushita Y, Marui H, Anzai T, Hashiguchi M, and Mochizuki M. 2015. Effect of database profile variation on drug safety assessment: an analysis of spontaneous adverse event reports of Japanese cases. Drug Des Devel Ther 9:3031-3041. 10.2147/DDDT.S81998

PMDA. 2015. Pharmaceuticals and Medical Devices Agency, Japan. Available at https://www.pmda.go.jp/english/index.html.

R Core Team. 2015. R: A Language and Environment for Statistical Computing. Vienna, Austria.

Raschi E, Piccinni C, Poluzzi E, Marchesini G, and De Ponti F. 2013. The association of pancreatitis with antidiabetic drug use: gaining insight through the FDA pharmacovigilance database. Acta Diabetol 50:569-577. 10.1007/s00592-011-03407

SQLite Development Team. 2015. SQLite. 